\title{
Stratifications of equivariant varieties
}

\author{
M.J. Field
}

\begin{abstract}
Let $G$ be a compact Lie group and $V$ and $W$ be linear $G$ spaces. A study is made of the canonical stratification of some algebraic varieties that arise naturally in the theory of $c^{\infty}$ equivariant maps from $V$ to $W$. The main corollary of our results is the equivalence of Bierstone's concept of "equivariant general position" with our own of "G transversal". The paper concludes with a description of Bierstone's higher order conditions for equivariant maps in the framework of equisingularity sequences.
\end{abstract}

Let $G$ be a compact lie group and $V$ and $W$ be linear $G$ spaces (that is, $G$ representations). A map $f: V \rightarrow W$ is said to be equivariant if $f(g v)=g f(v)$ for all $g \in G$ and $v \in V$. In this paper we study the stratifications of some algebraic varieties that arise naturally in the theory of $C^{\infty}$ equivariant maps from $V$ to $W$. As corollaries of our results we obtain the equivalence of Bierstone's definition of "equivariant general position" [1], and our definition of "G transversal" $[3,4]$. We also obtain a reformulation of Bierstone's higher order conditions described in [2].

\section{Stratifications}

We start by recalling some facts about stratifications of real algebraic sets (for more details we refer to Mather's exposition in [6]).

Received 26 November 1976. 
Let $X$ be an algebraic subset of $R^{m}$. A stratification of $X$ is a locally finite partition of $X$ into connected submanifolds of $R^{m}$, the strata of $X$, such that the frontier (in $R^{m}$ ) of each stratum is a union of lower dimensional strata. For $0 \leq j \leq m$, we define $x_{j}$ to be the union of all strata of dimension $j$ and refer to $x_{j}$ as the $j$-dimensional stratum of $X$. Whilst $X_{j}$ is a submanifold of $R^{m}$ of dimension $j$ (possibly empty), it need not be connected nor need the frontier of $X_{j}$ be a union of $X_{k}$ 's, $k<j$. In the sequel, we shall always regard the stratification of $X$ as consisting of the set of $j$-dimensional strata of $X, 0 \leq j \leq m$, and we shall denote the stratification of $X$ by $\left\{x_{j}\right\}$. We say that the stratification is an $\alpha$-stratification, where $\alpha$ may be $c^{\infty}$, analytic, semi-algebraic, and so on, if the strata are all of class $\alpha$. The stratification is termed Whitney regular if, given any pair $\left(x_{j}, x_{k}\right)$, with $j<k$, Whitney's regularity condition (b) holds at every point $x$ of $x_{j} \cap \overline{X_{k}}$ :

If $\left\{p_{i}\right\}$ and $\left\{q_{i}\right\}$ are sequences of points of $X_{j}$ and $X_{k}$ respectively such that both sequences converge to $x$, the line joining $p_{i}$ to $q_{i}$ converges to a line $L$, and the tangent space $T_{q_{i}} X_{k}$ converges in the grassmannian of $k$-dimensional subspaces of $R^{m}$ to $T$, then $T \supset L$.

A minimal a-stratification of $X$ is a Whitney stratification $\left\{x_{j}\right\}$ of $X$ such that if $\left\{Y_{j}\right\}$ is any other Whitney a-stratification of $X$, then either the two stratifications are the same or there exists $j_{0}$ such that $X_{j}=Y_{j}, j>j_{0}$, and $X_{j_{0}} \supset Y_{j_{0}} \cdot$ Clearly if a minimal $\alpha$-stratification of $X$ exists, it is unique. As described in [6], $X$ has a minimal semi-algebraic stratification - the so called canonical stratification of $X$. This stratification is also the minimal $C^{\infty}$ and (strongly) analytic stratification of $X$.

Suppose that $V$ and $W$ are finite dimensional vector spaces and that 
we are given a polynomial map

$$
P: V \times R^{k} \rightarrow W
$$

Let $X=P^{-1}(0)$ and, for each $t \in R^{k}$, set $X_{t}=\{x \in V: P(x, t)=0\}$. We are interested in the family of germs of $x_{t}$ at zero as $t$ varies in $R^{k}$ (cf. Varčenko [9]). Giving $X$ the canonical stratification $\left\{x_{j}\right\}$, we define a Whitney stratification of $V \times R^{k}$ by taking as top dimensional stratum $\left(V \times R^{k}\right) \backslash X$ and $\left\{X_{j}\right\}$ for the remaining strata. It is convenient to continue to use the notation $\left\{x_{j}\right\}$ for this stratification of $V \times R^{k}$ and we shall do this in the sequel. Finally, we shall regard $k^{k}$ as embedded in $V \times R^{k}$ as $\{0\} \times R^{k}$. We recall from [4].

DEFINITION. An equisingularity sequence for the family $\left\{x_{t}: t \in R^{k}\right\}$ is a Whitney stratification $\left\{\Lambda_{i}\right\}$ of $R^{k}$ such that

$$
\left\{x_{j} \backslash R^{k}, \Lambda_{i}: 0 \leq j \leq k+\operatorname{dim}(V), 0 \leq i \leq k\right\}
$$

is a Whitney stratification of $X \cup k^{k}$ and $x_{j} \cap \Lambda_{i}$ is empty for $j<i$, and an open, possibly empty, subset of $\Lambda_{i}$ for $j=i$.

DEFINITION. We say that the equisingularity sequence $\left\{\Lambda_{i}\right\}$ is a fundamental equisingularity sequence for $\left\{x_{t}: t \in R^{k}\right\}$ if it is minimal amongst the set of semi-algebraic equisingularity sequences for $\left\{x_{t}: t \in R^{k}\right\}$.

REMARKS. 1. The methods of [4] show that a fundamental equisingularity sequence for $\left\{x_{t}: t \in R^{k}\right\}$ exists and is in fact minimal amongst $C^{\infty}$ and strongly analytic equisingularity sequences. Since it is clearly unique we shall in future refer to it as the fundamental equisingularity sequence of $\left\{x_{t}: t \in k^{k}\right\}$. In the next section we shall 
give a rather explicit characterization of fundamental equisingularity sequences for a class of algebraic sets arising in the theory of equivariant maps.

2. In [4], an equisingularity sequence was defined to be a sequence $R^{k}=A_{0} \supseteq A_{1} \supseteq \cdots \supseteq A_{k}$ such that the sets $\Lambda_{k-i}=A_{i} \backslash A_{i+1}$ satisfied the first definition above. In other words, it was defined in terms of the filtration by codimension of the stratification of $R^{k}$.

3. The sets $\Lambda_{j}$ give a measure of the degree of Whitney equisingularity of the family $\left\{X_{t}: t \in R^{k}\right\}$. For example, it follows from Thom's First Isotopy Lemma that $\left\{\operatorname{germ}\left(X_{t}\right)\right.$ at zero $\left.: t \in R^{k}\right\}$ is locally topologically trivial over $\Lambda_{k}$.

We conclude this section with some remarks about the pullback of a Whitney stratification. Let $P: R^{m} \times R^{n} \rightarrow R^{n}$ be a $C^{\infty}$ map. We say that $P$ is a projection map if there exists a $C^{\infty}$ diffeomorphism $h$ of $R^{m} \times R^{n}$ such that the composite $P h$ is the projection of $R^{m} \times R^{n}$ on $R^{n}$. In case $P$ is also a polynomial, we say that $P$ is an algebraic projection map. We have the following trivial

PROPOSITION. Let $P: R^{m} \times R^{n} \rightarrow R^{n}$ be an algebraic projection map and $X$ be an algebraic subset of $R^{n}$ with canonical stratification $\left\{X_{, j}\right\}$. Then $\left\{P^{-1} X_{j}\right\}$ is the canonical stratification of the algebraic set $P^{-1} X$

REMARK. If we had only required $P$ to be a submersion, it would follow from $\left[6\right.$, Corollary 8.8] that $\left\{P^{-1} X_{j}\right\}$ is a Whitney stratification on $P^{-1} X$. However, it is easy to find examples showing that it need not be minimal.

\section{Varieties defined by equivariant maps}

Let $G$ be a compact lie group and $V$ and $W$ be linear G-spaces. 
We let $P_{G}(V, W)$ denote the space of equivariant polynomial maps from $V$ to $W$ and $P_{G}(V)$ denote the space of real valued $G$-invariant polynomials on $V$. As is well known, the compactness of $G$ implies that $P_{G}(V, W)$ is a finitely generated $P_{G}(V)$-module and $P_{G}(V)$ is finitely generated as an algebra (see, for example, [7]).

For the remainder of this paper we shall assume that $V$ has no proper subspaces on which $G$ acts trivially (that is, $V$ contains no trivial $G$ representations).

Let $F_{1}, \ldots, F_{s} \in P_{G}(V, W)$ and define $F: V \times R^{s} \rightarrow W$ by

$$
F(x, t)=\sum_{j=1}^{\varepsilon} t_{j} F_{j}(x),
$$

where $t=\left(t_{1}, \ldots, t_{s}\right) \in R^{s}$ and $x \in V$. Clearly $F \in P_{G}\left(V \times R^{s}, W\right)$, where we have given $V \times R^{S}$ the G-action defined by taking the product of the given action on $V$ with the trivial action on $R^{s}$.

Set $X=F^{-1}(0)$ and let $\left\{X_{j}\right\}$ denote the canonical stratification of $X$ (the top dimensional stratum is, by the convention of Section 1 , $\left.\left(V \times R^{S}\right) \backslash X\right)$. The minimality of the stratification implies that each stratum is a G-invariant submanifold of $V \times R^{s}$. Let $\Pi: V \times R^{s} \rightarrow R^{s}$ denote the projection on $R^{B}$ and define the family $\left\{X_{t}^{\prime}: t \in R^{s}\right\}$ by setting $X_{t}=\pi^{-1}(t) \cap X=\{x \in V: F(x, t)=0\}$.

One further piece of notation before we state our main result. If $Y$ is a connected analytic subset of $R^{S}$ we define

$$
\begin{aligned}
\operatorname{reg}_{i} Y & =\emptyset, i \neq \operatorname{dim}(Y) \\
& =\text { regular part of } Y, i=\operatorname{dim}(Y) .
\end{aligned}
$$

More generally, if $U X_{\alpha}$ is the decomposition of an analytic subset $X$ of $R^{s}$ into its connected components, we define 


$$
\operatorname{Reg}_{i} X=\underset{\alpha}{U} \operatorname{reg}_{i} X_{\alpha}
$$

THEOREM. A fundamental equisingularity sequence $\left\{\Lambda_{i}\right\}$ for $\left\{X_{t}: t \in R^{s}\right\}$ exists. It is characterised by any of the following equivalent conditions:

1. $\Lambda_{i}=\underset{j}{U} \operatorname{reg}_{i}\left(X_{j} \cap R^{s}\right), 0 \leq i \leq s ;$

2. if $t \in X_{j} \cap R^{s}$, then $t \in \Lambda_{i}$ if and only if $\operatorname{dim}\left(T_{t} X_{j} \cap R^{s}\right)=i ;$

3. if $t \in X_{j} \cap R^{s}$, then $t \in \Lambda_{i}$ if and only if $\operatorname{dim}\left(\pi\left(T_{t} X_{j}\right)\right)=i$.

Finally suppose $t \in \Lambda_{i} \cap X_{j}$. Then the connected component of $X_{j} \cap R^{s}$ containing $t$ is an open subset of $\Lambda_{i}$.

REMARK. We must emphasise that this theorem is special to the class of equivariant sets that we are considering. None of the statements of the theorem holds for arbitrary varieties.

Before starting the proof of the theorem we shall prove two lemmas.

LEMMA 1. Suppose $U^{\cdot}$ is a G-invariant linear subspace of $V \times R^{s}$. Then

$$
U=(V \cap U) \oplus\left(R^{s} \cap U\right)
$$

In particular,

$$
U \cap R^{S}=\Pi(U) .
$$

Proof. $U$ has a unique decomposition $A \oplus B$, where $A$ is a sum of (non-trivial) irreducible G-representations and $B$ is a trivial representation. Since $V$ does not contain any trivial representation, $A \subset V$ and $B \subset R^{S}$. // 
LEMMA 2. Suppose $t \in R^{S} \cap X_{j}$ and $\operatorname{dim} T_{t} X_{j} \cap R^{s}=i$. Then the connected component of $X_{j} \cap R^{s}$ containing $t$ is a submanifold of $R^{s}$ of dimension $i$.

Proof. Let $A$ denote the connected component of $X_{j}$ containing $t$ and $B$ denote the component of $A \cap R^{S}$ containing $t$. By Lemma 1 , $T_{b} A \cap R^{s}=\Pi\left(T_{b} A\right)$ for $b \in B$ and so a simple semi-continuity argument shows that $\operatorname{dim}\left(T_{b} A \cap R^{s}\right)=i$, for all $b \in B$.

$A$ is a G-invariant submanifold of $V \times R^{S}$ and so, given $b \in B$, there exists an open neighbourhood $U$ of $b$ in $V \times R^{s}$ and a $C^{\infty}$ equivariant diffeomorphism $h: U \rightarrow h(U) \subset V \times R^{s}$ which maps $A \cap U$ onto an open neighbourhood of zero in $T_{b} A$. Since $h$ is equivariant, $h\left(R^{S} \cap U\right) \subset R^{S}$ and so $h$ maps $A \cap U \cap R^{S}$ onto an open neighbourhood of zero in $T_{b} A \cap R^{S}$. Hence $B$ is a submanifold of $R^{S}$ of dimension $i . / /$

Proof of Theorem. Lemma 1 implies Conditions 2 and 3 are equivalent; Lemma 2 implies Conditions 1 and 2 are equivalent. We claim that $\left\{\Lambda_{i}\right\}$ is an equisingularity sequence. Condition $I$ implies that $X_{j} \cap \Lambda_{i}$ is empty for $j<i$ and an open subset of $\Lambda_{i}$ for $j=i$. It remains to be checked that $\left\{X_{j} \backslash R^{s}, \Lambda_{i}\right\}$ is a Whitney stratification of $X \cup R^{s}$. Now $\left(X_{j} \backslash R^{s}, X_{k} \backslash R^{s}\right), j<k$, and $\left(\Lambda_{j}, R^{s} \backslash X_{i}\right), j<i$, satisfy Whitney's regularity condition (b) where applicable since the stratification $\left\{X_{j}\right\}$ is Whitney. Let $t \in \Lambda_{j} \cap \bar{\Lambda}_{k}, k>j$. We must verify that condition (b) holds at $t$ for the pair $\left(\Lambda_{j}, \Lambda_{k}\right)$. Let $p_{i} \rightarrow t, q_{i} \rightarrow t$ be sequences of points of $\Lambda_{j}$ and $\Lambda_{k}$ respectively such that the line joining $p_{i}$ to $q_{i}$ converges to $L$ and $T_{q_{i}} \Lambda_{k}$ converges to $T$, where $T$ is a $k$-dimensional subspace of $R^{\mathcal{S}}$. Taking subsequences if necessary, we may 
suppose that $p_{i} \in X_{\alpha}, q_{i} \in X_{\beta}$, for some fixed $\alpha$ and $\beta$, and that $T_{q_{i}} X_{B}$ converges to a $\beta$-dimensional subspace $\tilde{T}$ of $V \times R^{S}$. Now in general $\tilde{T}$ will not contain $T$ and all that we can say is $\Pi(\tilde{T})=T$. But since our stratification is $G$-invariant and $V$ does not contain any trivial representation, we may apply Lemma 1 to deduce that $\tilde{T} \cap R^{\mathcal{E}}=T$. Since $\left\{X_{j}\right\}$ is Whitney regular, $\tilde{T} \supset L$. But $L \subset R$ and so $T \supset L$. Since $\left(X_{i} \backslash R^{s}\right) \cap \bar{\Lambda}_{j}=\varnothing$, for all $i$ and $j$, condition (b) is vacuously satisfied for the pair $\left(X_{i} \backslash R^{S}, \Lambda_{j}\right\}$. As the frontier conditions clearly hold, it follows that $\left\{X_{j} \backslash R^{s}, \Lambda_{i}\right\}$ is a Whitney stratification of $X \cup R^{S}$. Finally we remark that the equisingularity sequence that we have constructed is obviously minimal and so the fundamental equisingularity sequence of $\left\{X_{t}: t \in R^{s}\right\}$. //

REMARK* . If, following $[3,4]$, we define $A_{j}=\underset{i \leq s-j}{U} \Lambda_{i}, 1 \leq j \leq s$, it is easy to verify that $A_{j}$ is a closed semi-algebraic cone, vertex origin. It is natural to ask whether the sets $A_{j}$ are projective algebraic varieties or, what amounts to the same thing, if they are analytic cones. Indeed, if we had taken the stratification of $X$ defined by taking the real part of the canonical complex analytic stratification of the complexification of $X$ the equisingularity sequence that we obtain does give projective algebraic subsets. However, this stratification need not coincide with the canonical stratification. For example, the stratifications differ for the varieties defined by $x\left(x^{2}+y^{2}\right)$ or $y^{2}+z^{2} x^{3}+x^{5}$. In any case, we ask: Are the varieties $A_{j}$ projective algebraic?

Continuing with the notation and assumptions of the theorem, suppose we are given polynomials $p_{k j} \in P_{G}(V)$, where $1 \leq i \leq s$ and, for each

* I am grateful to T.C. Kuo for pointing out these examples to me. 
$i, 1 \leq j \leq m_{i}$. We shall assume that $p_{i j}(0)=0$ for all $i$ and $j$ (since $V$ contains no trivial representations, this implies that $D p_{i j}$ vanishes at zero for all $i$ and $j$ ).

Set $m=s+\sum_{i=1}^{s} m_{i}$ and give $R^{m}$ the coordinates $\left(t_{1}, t_{11}, \ldots, t_{1 m_{1}} ; t_{2}, t_{21}, \ldots, t_{s m_{s}}\right)$. Let $p: R^{m} \rightarrow R^{s}$ be the obvious projection map. We also use the notation $p$ for the projection $(\operatorname{Id} \times p): V \times R^{m} \rightarrow V \times R^{s}$. Define $Q: V \times R^{m} \rightarrow W$ by

$$
Q(x, t)=\sum_{i=1}^{s}\left(t_{i}+\sum_{j=1}^{m_{i}} t_{i j} p_{i j}(x)\right) F_{i}(x) \text {. }
$$

$Q$ is equivariant if we give $V \times R^{m}$ the $G$ action defined by taking the product of the given action on $V$ with the trivial action on $R^{m}$. Denote the fundamental equisingularity sequence associated to the variety defined by $Q$ by $\left\{\Sigma_{i}\right\}$.

PROPOSITION.

$$
\begin{aligned}
\Sigma_{i} & =p^{-1} \Lambda_{i}, \quad i=m, \ldots, m-s \\
& =\emptyset, \quad i<m-s .
\end{aligned}
$$

Proof. We claim that the map $\tilde{p}: V \times R^{m} \rightarrow V \times R^{\Theta}$ defined by

$$
\tilde{p}\left(x, t_{i}, t_{i j}\right)=\left(x, t_{i}+\sum_{j=1}^{m_{i}} t_{i j} p_{i j}(x)\right)
$$

is a projection map in the sense of section 1. Indeed the map $h: V \times R^{m} \rightarrow V \times R^{m}$ defined by

$$
h\left(x, t_{i}, t_{i j}\right)=\left(x, t_{i}-\sum_{j=1}^{m_{i}} t_{i j} p_{i j}(x), t_{i j}\right)
$$

is a $C^{\infty}$ equivariant diffeomorphism and $\tilde{p} h=p$.

Applying the proposition at the end of Section 1, we see that the pull- 
back of the canonical stratification of $X$ by $\tilde{p}$ gives the canonical stratification of $Q^{-1}(0)$. The characterisation of a fundamental equisingularity sequence given by Condition 1 of the theorem implies that the fundamental equisingularity sequence for $Q^{-1}(0)$ is given by $\left\{\tilde{p}^{-1}\left(\Lambda_{i}\right)\right\}=\left\{p^{-1}\left(\Lambda_{i}\right)\right\}$

\section{Applications to the theory of equivariant maps}

Let $C_{G}^{\infty}(V, W)$ denote the space of $C^{\infty}$ equivariant maps from $V$ to $W$ and $C_{G}^{\infty}(V)$ denote the space of $C^{\infty}$ equivariant real valued maps on $V$. $C_{G}^{\infty}(V, W)$ has the structure of a $C_{G}^{\infty}(V)$-module. Fix a minimal set of homogeneous polynomials which generate $P_{G}(V, W)$ as a $P_{G}(V)$-module, say $\left\{F_{1}, \ldots, F_{k}\right\}$. Then $\left\{F_{1}, \ldots, F_{k}\right\}$ generate $C_{G}^{\infty}(V, W)$ as a $C_{G}^{\infty}(V)$ module (for a proof of this non-trivial fact, see $[1,4,7]$ ). Moreover, if $R^{\mu}$ is a trivial G-space, $\left\{F_{1}, \ldots, F_{k}\right\}$ also generate $C_{G}^{\infty}\left(V \times R^{\mu}, W\right)$ as a $C_{G}^{\infty}\left(V \times R^{\mu}\right)$-module. Let $\left\{X_{j}\right\}$ denote the canonical stratification of the variety $X \subset V \times R^{k}$ defined by the polynomial $E(x, t)=\sum t_{j} F_{j}(x)$ and $\left\{\Lambda_{i}\right\}$ denote the corresponding fundamental equisingularity sequence. We shall briefly review two definitions of equivariant transversality. Full details are given in $[1,4]$. Let $f \in C_{G}^{\infty}\left(V \times R^{\mu}, W\right)$. Then there exist $f_{j} \in C_{G}^{\infty}\left(V \times R^{\mu}\right)$ such that

$$
f(x, u)=\sum_{j=1}^{k} f_{j}(x, u) F_{j}(x) .
$$

Whilst the maps $f_{j}$ are not necessarily uniquely determined by $f$, the $\operatorname{map} \gamma_{f}: R^{u} \rightarrow R^{k}$ defined by $\gamma_{f}(u)=\left(f_{I}(0, u), \ldots, f_{k}(0, u)\right)$ depends only on $f$ and the choice of generators $\left\{F_{1}, \ldots, F_{k}\right\}$. We let $G_{f}: V \times R^{\mathcal{u}} \rightarrow V \times R^{k}$ denote the "graph" map defined by 
$G_{f}(x, u)=\left(x, f_{1}(x, u), \ldots, f_{k}(x, u)\right)$. Both $\gamma_{f}$ and $G_{f}$ are $c^{\infty}$.

DEFINITION A (adapted from Bierstone [1]). Let $f \in C_{G}^{\infty}\left(V \times R^{\mu}, w\right)$. $f$ is said to be in equivariant general position to $0 \in W$ at $(0,0) \in V \times R^{k}$ if either $f(0,0) \neq 0$ or $f(0,0)=0$ and the map $G_{f}: V \times K^{\mathcal{H}} \rightarrow V \times R^{\mathcal{k}}$ is transversal to the canonical stratification of $x$ at zero.

DEFINITION B $([4])$. Let $f \in C_{G}^{\infty}\left(V \times R^{\mu}, W\right) \cdot f$ is said to be $G$-transversal to $0 \in W$ at $(0,0) \in V \times R^{k}$ if either $f(0,0) \neq 0$ or $f(0,0)=0$ and $\gamma_{f}: R^{\mu} \rightarrow R^{k}$ is transversal to the fundamental equisingularity sequence $\left\{\Lambda_{i}\right\}$ at zero.

We refer to [6] for the definition and basic properties of maps transversal to a Whitney stratification.

PROPOSITION. Definitions $\mathrm{A}$ and $\mathrm{B}$ are equivalent.

Proof. Let $f \in C_{G}^{\infty}\left(V \times R^{\mu}, W\right)$ and suppose $f(0,0)=0$. Let $t=\gamma_{f}(0) \in \Lambda_{i}$ and $G_{f}(0,0) \in X_{j}$. Now if $f$ is in equivariant general position to $(0,0)$ at $0, T_{t} X_{j}$ complements $\left(T(0,0)_{f}\right)\left(V \times R^{u}\right)$ in $V \times R^{k}$. Since $G_{f}$ is the identity on $V \times\{0\}$, this is equivalent to $\left(T_{0} \gamma_{f}\right)\left(R^{\mathcal{\mu}}\right)$ complementing $\pi\left(T_{t} X_{j}\right)$ in $R^{k}$. By Condition 3 of our theorem characterising fundamental equisingularity sequences, this is equivalent to $\left(T_{0} \gamma_{f}\right)\left(R^{\mathcal{u}}\right)$ complementing $T_{t} \Lambda_{i}$ in $R^{k}$. But this is just the condition for G-transversality. //

REMARKS. 1. We have put the definition of equivariant general position in a form that allows us to prove its equivalence with G-transversality with the least effort. In [1], the requirement that $V$ does not contain any trivial representations is dropped. The definition is then naturally stated as the transversality of the graph map $x \mapsto\left(x, f_{1}(x), \ldots, f_{k}(x)\right)$ to the canonical stratification of the variety 
associated to a minimal set of homogeneous generators of ${ }_{P_{G}}(V, W)$.

2. Since equivariant general position is an open condition [1], the proposition implies the opennes of $G$-transversality, answering a question raised in [4].

For the remainder of this section we intend to reformulate Bierstone's higher order equivariant general position conditions described in [2] in the framework of equisingularity sequences.

As Bierstone points out by an example in [1], although Definitions A and $B$ are quite satisfactory from the point of view of stability of intersections, the maps that can occur are sometimes undesirably singular.

EXAMPLE. Consider the space of $C^{\infty} S^{1}$ invariant maps from $C^{2}$ to $C^{2}$, where the $S^{1}$ action on the domain is multiplication by $\left(e^{3 i \theta}, e^{4 i \theta}\right)$ and on the target is multiplication by $\left(e^{10 i \theta}, e^{1 l i \theta}\right)$. A minimal set of homogeneous generators for $P_{S^{1}}\left(c^{2}, c^{2}\right)$ is $\left\{\left(z_{1}^{2} z_{2}, 0\right),\left(0, z_{1} z_{2}^{2}\right)\right\}$ and the corresponding fundamental equisingularity sequence is $\Lambda_{4}=\left\{\left(t_{1}, t_{2}\right) \in c^{2}:\left(t_{1}, t_{2}\right) \neq(0,0)\right\} ;$

$$
\Lambda_{3}=\Lambda_{2}=\Lambda_{1}=\varnothing ; \Lambda_{0}=\{(0,0)\} .
$$

(The subscripts refer to real dimension.) For $(t, s) \in C^{2}$, let $f_{t, s}\left(z_{1}, z_{2}\right)=\left(t z_{1}^{2} z_{2}, s z_{1} z_{2}^{2}\right) \cdot f_{t, s}$ is $C^{\infty}$ and $s^{1}$ invariant and is $S^{l}$ transversal to zero at zero provided both $t$ and $s$ are not zero. However, the maps $f_{t, 0}$ and $f_{0, s}$ are easily seen to be highly singular at zero by comparison with $f_{t, s}, t \neq 0, s \neq 0$. For instance, $D f_{t, s}\left(z_{1}, z_{2}\right)$ is a linear isomorphism off the $z_{1}$ and $z_{2}$ axes if $t$ and $s$ are non-zero. It is desirable that the transversality definition exclude the cases when either $s$ or $t$ is zero.

We make no attempt here to give a full description of the higher order theory developed by Bierstone to overcome this deficiency in the definition as a full presentation is given in his paper [2]. Instead we confine ourselfes to outlining the theory to a point where we can state our 
formulation of the higher order conditions.

Let $\left\{p_{1}, \ldots, p_{1}\right\}$ denote a minimal set of homogeneous algebra generators for the $R$-algebra $P_{G}(V)$. If we set

$$
P=\left(p_{1}, \ldots, p_{1}\right): V \rightarrow R^{I}
$$

and let $P^{*}: P\left(R^{1}\right)+P_{G}(V)$ denote the map defined by $P^{*}(q)=q P$, $q \in P\left(R^{l}\right)$, then $P^{*}$ is surjective (see, for example, [7]).

Schwarz' theorem [8], states that $P^{*}: C^{\infty}\left(R^{l}\right) \rightarrow C_{G}^{\infty}(V)$ is also surjective. That is, given $f \in C_{G}^{\infty}(V)$, there exists $g \in C^{\infty}\left(R^{l}\right)$ such that $f=g P$.

If $G$ acts trivially on $R^{\mathcal{H}}$, then $\left\{p_{1}, \ldots, p_{1}, t_{1}, \ldots, t_{u}\right\}$ is a minimal set of homogeneous generators for $P_{G}\left(V \times R^{\mu}\right)$ and so, given $h \in C_{G}^{\infty}\left(V \times R^{\mathcal{U}}\right)$, there exists $g \in C^{\infty}\left(R^{l} \times R^{\mathcal{U}}\right)$ such that $h(x, t)=g(P(x), t)$. Consequently, any $f \in C_{G}^{\infty}\left(V \times R^{u}, W\right)$ may be written, not necessarily uniquely, in the form

$$
f(x, t)=\sum_{j=1}^{k} g_{j}(P(x), t) F_{j}(x),
$$

$g_{j} \in C_{G}^{\infty}\left(R^{l} \times R^{\mathcal{u}}\right)$. The higher order conditions described by Bierstone come by differentiating this equation.

We use the notation of [5] to describe jets and jet spaces. Thus, if $f \in C^{\infty}(v, W)$, we let $j^{d} f(x)=\left(f(x), D f(x), \ldots, D^{d} f(x)\right)$ denote the $d$-jet of $f$ at $x, d=0,1, \ldots$. We denote the corresponding jet space by $f^{d}(V, W)$ and recall that

$$
f(V, W)=\underset{i=0}{\oplus} L_{s}^{i}(V ; W),
$$


where $L_{s}^{i}(V ; W)$ is the space of $i$-linear symmetric maps from $V$ to $W$. Define $Q: V \times R^{\mu} \rightarrow R^{1} \times R^{\mu}$ by $Q(x, t)=(P(x), t)$. $D Q: V \times R^{\mathcal{u}} \rightarrow L\left(V \times R^{\mathcal{u}}, R^{l} \times R^{\mathcal{u}}\right)$ and $D Q(x, t)=(D P(x), I d)$. Hence $D Q$ depends only on $x$, and we shall write $D Q(x)$ for $D Q(x, t)$ in the sequel.

Before we proceed further we need to recall some linear algebra. Suppose $E$ and $F$ are vector spaces and that we are given positive integers $m_{1}, \ldots, m_{s} ; q_{1}, \ldots, q_{s}$. Set $p=m_{1} q_{1}+\ldots+m_{s} q_{s}$ and $q=q_{1}+\ldots+q_{s}$. We define the "symetrization" map

$$
\text { symm }^{*}: \underset{j=1}{s}\left(L_{s}^{m} j(E ; F)\right)^{q} j+L_{s}^{p}\left(E ; X^{q} F\right)
$$

by

$$
\begin{aligned}
& \operatorname{symm}^{*}\left(\left(A_{1}\right), \ldots,\left(A_{s}\right)\right)\left(e_{1}, \ldots, e_{p}\right)= \\
& 1 /\left(q_{1} ! \ldots q_{s} !\right) \sum\left(A_{1}^{\perp}\left(e_{\sigma(1)}, \ldots, e_{\sigma\left(m_{1}\right)}\right),\right. \\
& \left.\ldots, A_{s}^{m_{s}}\left(e_{\sigma\left(p-m_{s}+1\right)}, \ldots, e_{\sigma(p)}\right)\right) \text {, }
\end{aligned}
$$

where $\left(e_{1}, \ldots, e_{p}\right) \in X^{p_{E}},\left(A_{j}\right)=\left(A_{j}^{1}, \ldots, A_{j}^{q} j\right) \in\left(L_{s}^{m_{j}}(E ; F)\right)^{q_{j}}$, and the sum is taken over all permutations $\sigma$ of $\{1, \ldots, p\}$ such that, if we set $M_{i j}=m_{1} q_{1}+\ldots+m_{j-1} q_{j-1}+i m_{j}$, $\sigma\left(M_{i j}+1\right)<\sigma\left(M_{i j}+2\right)<\ldots<\sigma\left(M_{i j}+m_{j}\right)$,

$$
j=1, \ldots, s-1, i=0, \ldots, q_{j}-1 .
$$

For $r=0,1, \ldots$ we define

$$
T_{r}^{\mu}: V \times \underset{i=0}{\oplus} L_{s}^{i}\left(R^{1} \times R^{\mu} ; R^{k}\right) \rightarrow L_{s}^{r}\left(V \times R^{\mu} ; R^{k}\right)
$$

by 


$$
T_{p}^{\mu}(x, a)=\sum_{s=1}^{r} \sum_{m_{1} q_{1}+\ldots+m_{s} q_{s}=r} a_{q}\left[\operatorname{symm}^{*}\left(\left(D^{m}{ }^{m}\right)^{q_{1}}, \ldots,\left(D^{m} s^{q_{s}}\right)(x)\right],\right.
$$

where we have set $q=q_{1}+\ldots+q_{s}$ and

$$
a=\left(a_{0}, \ldots, a_{p}\right) \in \underset{i=0}{r} L_{s}^{i}\left(R^{1} \times R^{\mu} ; R^{k}\right)
$$

(This rather complicated expression becomes the formula for the rth derivative of the map $g Q$ if we replace $a_{q}$ everywhere by $D^{q} g(Q(x))$.)

For $d=0,1, \ldots$ we define the equivariant polynomials

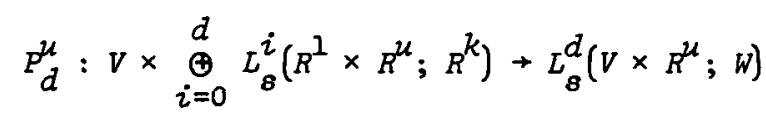

by

$$
P_{d}^{\mu}(x, a)=\sum_{r=0}^{d} \sum_{i=1}^{k}\left(\begin{array}{l}
d \\
p
\end{array}\right) T_{p}^{\mu}(x, a){ }_{i} \odot D^{d-r_{F}(x)}
$$

where " $O$ " denotes the symmetric tensor product and the subscript $i$ on $T_{p}^{\mu}(x, a)$ denotes the $i$ th component of $T_{r}^{\mu}(x, a)$ in $R^{k}$.

Setting $U_{d}^{\mu}=\left[P_{0}^{\mu}, \ldots, P_{d}^{\mu}\right]$, we see that

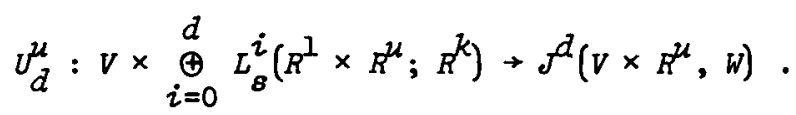

Let $x_{d}^{\mu}$ be the zero set of $u_{d}^{\mu}$ and $\left\{\Sigma_{d, i}^{u}\right\}$ denote the corresponding fundamental equisingularity sequence.

Let $f \in C_{G}^{\infty}\left(V \times R^{\mu}, W\right)$ and write

$$
f(x, t)=\sum_{i=1}^{k} g_{i}(P(x), t) F_{i}(x),
$$

$g_{i} \in C_{G}^{\infty}\left(R^{1} \times R^{\mu}\right)$. Set $g=\left(g_{1}, \ldots, g_{k}\right) \in C^{\infty}\left(R^{1} \times R^{\mu}, R^{k}\right)$ and define 


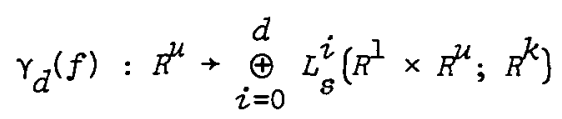

by

$$
\gamma_{d}(f)(t)=j^{d} g(0, t)=\left(g(0, t), \ldots, D^{d} g(0, t)\right) .
$$

DEFINITION (Bierstone [2]). Let $f \in C_{G}^{\infty}\left(V \times R^{\mathcal{u}}, w\right) . f$ is said to be in $d$ th approximation to equivariant general position to $0 \in W$ at $(0, t) \in V \times R^{\mathcal{u}}$ if either $f(0, t) \neq 0$ or $f(0, t)=0$ and $\gamma_{p}(f)$ is transversal to the fundamental equisingularity sequence. $\left\{\Sigma_{r, i}^{u}\right\}$ at $t$ for $0 \leq r \leq d$.

EXAMPLE. Following the notation of our previous example, the map $f_{t, s}$ is in $d$ th approximation to equivariant general position to $0 \in c^{2}$ at zero for all $d \geq 0$ if $t \neq 0$ and $s \neq 0$.

REMARKS. 1. It is easily shown that the above definition is equivalent to that stated in [2]. Moreover, it is shown in [2] that the definition is independent of the choice of $g$ implicit in the maps $\gamma_{d}$ and of the choice of generators $\left\{F_{1}, \ldots, F_{k}\right\}$ and $\left\{p_{1}, \ldots, p_{1}\right\}$. It is also shown that there exists $d_{0}$, depending on $V, W$, and $u$, such that $d_{0}$ th approximation to equivariant general position implies $d$ th approximation to equivariant general position for $d \geq d_{0}$. Equivariant general position is then redefined to be $d_{0}$ th approximation to equivariant general position.

2. It should be noted that no coefficients involving powers of the polynomials $\left\{p_{1}, \ldots, p_{1}\right\}$ appear in $U_{d}^{\mathcal{H}}$. The inclusion of such higher order terms has no effect on the fundamental equisingularity sequence - see the proposition at the end of section 2.

3. A natural question arising out of our formulation of the higher order transversality conditions is the extent to which the fundamental equisingularity sequence $\left\{\Sigma_{d, i}^{u}\right\}$ depends on $u$. For example, suppose 
$f \in C_{G}^{\infty}(V, W)$ is in equivariant general position to $0 \in W$ at $0 \in V$ and define $\tilde{f}: V \times R^{u} \rightarrow W$ by $\tilde{f}(x, t)=f(x)$. Is $\tilde{f}$ in equivariant general position to $0 \in W$ on $R^{u}$ ?

\section{References}

[1] Edward Bierstone, "General position of equivariant maps", Trans. Amer. Math. Soc. (to appear).

[2] Edward Bierstone, "Generic equivariant maps", submitted.

[3] Mike Field, "Transversalitê dans les G-variétés", C.R. Acad. Sci. Paris Sér. A 282 (1976), 115-117.

[4] Mike Field, "Transversality in G-manifolds", Trans. Amer. Math. Soc. (to appear).

[5] M. Golubitsky, V. Guillemin, Stable mappings and their singularities (Graduate Texts in Mathematics, 14. Springer-Verlag, New York, Heidelberg, Berlin, 1973).

[6] John N. Mather, "Stratifications and mappings", Dynamical systems, 195-232 (Proc. Sympos. University of Bahia, Salvador, Brasil, 1971. Academic Press [Harcourt Brace Jovanovich], New York and London, 1973).

[7] Valentin Poènaru, Singularités $C^{\infty}$ en présence de symétrie (Lecture Notes in Mathematics, 510. Springer-Verlag, Berlin, Heidelberg, New York, 1976).

[8] Gerald W. Schwarz, "Smooth functions invariant under the action of a compact Lie group", Topology 14 (1975), 63-68.

[9] A.N. Varčenko, "Theorems on the topological equisingularity of families of algebraic varieties and families of polynomial mappings", Math. USSR-Izv. 6 (1972), 949-1008.

Department of Pure Mathematics,

University of Sydney,

Sydney, New South Wales. 